\title{
Discussions with Leaders: A Conversation Between Richard Wahl and Johannes Czernin
}

\author{
Richard L. Wahl ${ }^{1}$ and Johannes Czernin ${ }^{2}$
}

${ }^{1}$ Mallinckrodt Institute of Radiology, Washington University School of Medicine, St. Louis, Missouri; and ${ }^{2}$ David Geffen School of Medicine at UCLA, Los Angeles, Californiai

$\mathbf{J}$ ohannes Czernin, editor in chief of The Journal of Nuclear Medicine, recently initiated a series of recorded discussions with leaders in nuclear medicine and molecular imaging. The second of these, conducted on October 23, 2018, is excerpted below. Richard L. Wahl is the Elizabeth E. Mallinckrodt Professor and head of radiology at Washington University School of Medicine in St. Louis, Missouri, director of the university's Mallinckrodt Institute of Radiology (MIR), and a professor of radiation oncology. Wahl's research played an important role in development of radioimmunotherapy for non-Hodgkin lymphoma. He has also been a pioneer in the use of PET scans to diagnose and assess treatment of a broad array of cancers and other diseases and remains at the forefront of efforts to combine quantitative data from PET scans with CT to help physicians more precisely diagnose and characterize cancers. Wahl and colleagues developed the PET Response Criteria in Solid Tumors (PERCIST) for assessing treatment response in cancer. He is an elected member of the National Academy of Medicine, holds 18 patents, and has published more than 400 peerreviewed scientific manuscripts. He is the primary author of several textbooks, including Principles and Practice of PET and PET/ $C T$. He has a strong interest in quantitative imaging, is on the coordinating committee of the Quantitative Imaging Biomarkers Alliance (QIBA) in the Radiological Society of North America (RSNA), and has been a lead investigator in the Quantitative Imaging Network (QIN) for the National Institutes of Health (NIH). In addition to numerous awards and honors, he was named in 2018 as the recipient of the SNMMI Georg Charles de Hevesy Nuclear Pioneer Award for his lifetime contributions to nuclear medicine.

Dr. Czernin: You currently serve as the Elizabeth E. Mallinckrodt Professor and head of radiology at Washington School of Medicine in Saint Louis. This is also where you attended medical school. How does it feel to return to your academic roots?

Dr. Wahl: This was my third move to St. Louis, so it is not an unfamiliar place. It feels good to be back-for 4 years now. I first moved to St. Louis from a small town in Iowa to attend a medical school reputed to train physician scientists. I then left Washington University for a brief stay at the University of California San Diego (UCSD) in internal medicine, thinking I'd become a cardiologist, before returning to Washington University for a second time to become a diagnostic radiologist and nuclear medicine physician. Washington University exposed me to the power of academic medicine and provided a great training ground during my formative academic years. This culture of innovation, along

COPYRIGHT $\odot 2019$ by the Society of Nuclear Medicine and Molecular Imaging. DOI: 10.2967/jnumed.118.223503 with large clinical and educational missions, is one I am happy to rejoin and build upon in my role directing MIR.

Dr. Czernin: You are a board-certified radiologist but developed an early interest in nuclear medicine. What attracted you to this field?

Dr. Wahl: During medical school, I developed a love for and abiding interest in both anatomy and physiology. Washington University and MIR had very early $\mathrm{CT}$ scanners for the brain

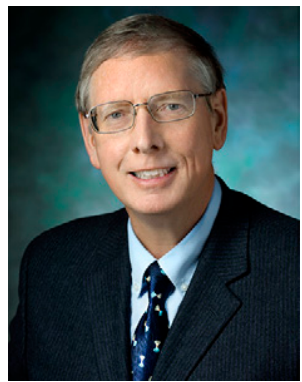

Richard L. Wahl, MD and whole body, and it was obvious this technology would revolutionize the practice of radiology. Nuclear medicine was my first rotation in my radiology residency after completing my internal medicine internship at UCSD. I found the field absolutely fascinating. You could use radioactivity and whole-body scans to evaluate different organs--often at the same time. I didn't have to be so focused on specializing in a single organ system (I was never very good at focusing on organ-specific areas). Studying physiology in the body from the top of the head to the bottom of the feet was very attractive and complementary but different from what I could do in the anatomic practice of radiology.

So I moved into a focused nuclear medicine academic practice as a career choice, in part because of this initial positive experience. I also had great role models at Washington University. It was a terrific research environment, with Michael Welch leading radiochemistry, Barry Siegel leading nuclear medicine, and Michel Ter-Pogossian giving us physics lectures. And, of course, Michael Phelps was there for 1 year of my medical school training; I did not know him at that time-although his legacy of functioning PET scanning equipment remained.

It was an exciting place. I think I was the first research resident ever at MIR. They gave me a grand total of 4 months to do research. We developed radiolabeled monoclonal antibody fragments for imaging and tested them in animal models. This short but intense research time, along with clinical practice, led me to nuclear medicine as a career.

After completing my training I was recruited as an assistant professor and codirector of general nuclear imaging by William $\mathrm{H}$. Beierwaltes at the University of Michigan, where I joined again a great research and clinical environment. Michigan was oriented toward therapy at that time, which was a huge plus for me, because this provided a substantial infrastructure and institutional knowledge in this area.

Dr. Czernin: Research is often not the focus of radiology departments, which are extremely high-volume clinical operations. Salaries often depend on patient numbers, with the relative value 
unit ( $R V U)$ model frequently used to define compensation. In contrast, nuclear medicine is a low-volume operation with great research potential. As the chair of a radiology department, how do you make this work business-wise? How can nuclear medicine remain financially and thus academically viable?

Dr. Wahl: This is an important question. I am responsible for making the entire radiology department budget work. We are a large radiology department--one of the largest in the world. We have the largest diagnostic radiology training program in the country. So we influence a lot of young minds, and certainly a substantial number of our trainees are choosing nuclear medicine as a field. But, as you know, the RVU model is established in radiology and hard to get around. You mentioned correctly that some nuclear medicine applications don't fit well with that model, although some aspects of our work do fit with the RVU model. PET/CT, for example, is becoming a high-volume examination. We need to work on achieving appropriate professional reimbursement levels. This will be very important to ensure that therapeutic nuclear medicine and more innovative diagnostic approaches are appropriately reimbursed in the future. Moreover, some very attractive new procedures are emerging (think theranostics with its huge clinical and economic potential), for which RVU-based models will need to be modified and adjusted.

Dr. Czernin: How do you deal with this at Washington University? You have a clinically and academically vibrant Division of Nuclear Medicine. How do you generate the funds to pay salaries and support the research program? Do you use discretionary funds or mainly research grants?

Dr. Wahl: There is no way around the fact that research costs money. Our research depends on grants, practice revenues, endowments, medicine? Training grants certainly help to free up time for physicians and scientists to receive additional research training. There has to be continued investment to move the field ahead. There needs to be continued effort to innovate in our practices.

Dr. Czernin: It is important for nuclear medicine to be financially independent. Look at the recent successes coming from Europe and Australia with the translation of prostate cancerand neuroendocrine tumor-targeted theranostics. Nuclear medicine is strong and independent in most of Europe and Australia, as well as in Asian countries. Many of these countries have what Americans might call socialized medicine. Clinical operations are based on societal value and not on RVUs, physicians' salaries are not volume based, and research is funded through entirely different mechanisms. This takes away a lot of the economic pressure and allows nuclear medicine to thrive. Although nuclear medicine is fiscally and administratively independent in these settings, it is highly collaborative. Programs interact closely with radiology but also with all the other disciplines, including radiation oncology, urology, endocrinology, and others. My questions are, as always: Why is it so different in the United States? Why has nuclear medicine become the stepchild of radiology? I believe that the answer is fairly simple: There is not much money to be made in nuclear medicine. There may be a great opportunity for this to change with the emergence of clinical theranostics. Another reason for the success of nuclear medicine in other countries is that they have far superior 5-year training programs, including crosssectional imaging and rotations in radiation oncology, oncology, cardiology, and other disciplines.

Dr. Wahl: In order to be an efficient health-care delivery system, a lot of collaboration is needed. I think that the current

\section{"With artificial intelligence and quantitation, I see radiology and nuclear medicine moving from qualitative and subjective tools to quantitative and objective ones."}

and philanthropy. One has to invest, and some of the investments we make are broadly in radiology, including nuclear medicine. Sometimes the investment returns come slowly. At MIR, we routinely invest in the more basic aspects of imaging science. We also think strategically about what kinds of investments we can make that will have real impacts on patient care and management. Radiology and nuclear medicine have to keep innovating, changing, and evolving. If we don't evolve, nuclear medicine risks being divided up among other specialties.

Radiology is a high-volume/low-margin business. You have to pay skilled professionals competitively. So funds are limited, and one has to be strategic about what to invest in. At our institution we are fortunate to have a long tradition of strength, particularly on the radioisotope production side, and we were fortunate recently to secure a P41 grant from NIH. This is a National Center of Cyclotron Excellence award that enables us to train physicians and scientists in cyclotron-produced pharmaceuticals.

One important issue in nuclear medicine is that we are potentially not training our students and residents as well as we must to preserve and advance the field. One could argue about how this should be done, but who will our innovators be and who are the ones who will identify innovation opportunities in nuclear vertical hierarchical model that creates silos will become more historical. The new model will be based on integration and collaboration among specialties to avoid redundancy. As you point out, the need for close-working relationships with other disciplines is incredibly important. You and I have both seen in the last couple of decades a major swing in revenue sources in nuclear medicine. A large proportion of professional revenues now come from PET/CT. Much should be coming from theranostics in the future. If each therapy cycle is reimbursed at more than $\$ 40,000$, then there should be financial funds flow models that allow nuclear medicine to be financially viable, similar to the models used in oncology. The financial margins for health-care systems on these therapies could be sufficient, if properly allocated, to easily support a significant clinical operation delivering service in nuclear medicine.

The devil is in the details, but a model different from RVUs for professional nuclear medicine services will almost certainly be required and will evolve. That's what a lot of multispecialty clinics are doing and where we are headed: a roll-up of technical and professional revenues to support the professional headcount needed to do proper clinical work and research.

Dr. Czernin: At the University of California, Los Angeles, we are currently planning and building an outpatient theranostics 
center. In addition to providing great patient care, this will also attract an entirely different type of nuclear medicine trainee who is more oriented toward medicine and oncology than radiology.

Dr. Wahl: Having cross-sectional imaging training is essential for success in nuclear medicine, given the major role of hybrid imaging. But I think there is a lot of room for nuclear medicine physicians to manage their therapeutic patients much in the same way that radiation oncologists manage their patients during radiation therapy. This represents a great opportunity for us to improve our training and expand our practice space. This is the area in which an extra couple of years' training really helps in some of the programs in Europe, Canada, and Australia, among other places. The precise training requirements for being first a treating physician and also a nuclear medicine physician remain to be defined. We will need to recruit those individuals who have an interest in this combination of diagnostics and therapeutics. If we don't, radiation oncologist or others will certainly step in to provide these services.

Dr. Czernin: I agree. If we don't take care of this, change the training, and take the lead in theranostics, then evolution will take care of nuclear medicine, at least in the United States.

Dr. Wahl: I am not sure exactly how long the training should be, but it should be sufficient to train for the complexities of therapy. Some of the curricula that we see-sort of "nuclear medicine lite" with an integrated nuclear medicine and radiology residency in a short period of time-may not be sufficient for complex nuclear medicine therapies as currently configured. Comprehensive training should be broader than just creating a diagnostic nuclear medicine expert. Perhaps dedicated fellowships in theranostics could be one solution. We have an aging nuclear medicine workforce that must be refreshed, meaning that we have challenging training needs for both nuclear medicine and radiology physicians. A potentially greater gap is on the therapeutic side, because this is an area that will evolve and expand quite quickly. Who is going to do it? It should be people who understand radiation biology, imaging, and therapy. Dosimetry will likely be important in some settings. It's difficult for me to believe that we will end up with a single training path that could fit all patients' needs.

Dr. Czernin: Let's switch gears a bit to look at your extraordinary contributions to the field. In a 1993 article from The Journal of Nuclear Medicine (J Nucl Med. 1993:34:1190-1197), you introduced the term "anatomometabolic" imaging, which foresaw the hybrid PET/CT imaging culture that emerged in the late 1990s with David Townsend's and Thomas Beyer's landmark article (J Nucl Med. 2000;41:1369-1979). In 2009, you introduced and published PERCIST 1.0 (J Nucl Med. 2009;50[suppl 1]:122S150S). As of today this paper has been cited 2,121 times. This had a tremendous impact on the field as early response assessment using ${ }^{18}$ F-FDG PET became an accepted consideration. What do you see as your most defining contribution?

Dr. Wahl: I appreciate your comments. I've had a number of contributions that came from being involved early on in projects that have led to clinical translation. As a physician, I am particularly focused on science that affects the lives of patients. Take, for example, radiolabeled monoclonal antibody targeting-I would say that the work on ${ }^{131}$ I anti-CD20 targeting to treat lymphoma conducted at Michigan with Mark Kaminski was a defining contribution. This was an attempt to develop precision medicine. It was, in fact, a theranostic concept with precision targeting, precision dosimetry, and a patient-specific dosing approach. This went all the way from preclinical research to U.S. Food and Drug Administration approval and benefited thousands of patients.

Dr. Czernin: This treatment was quite effective, but it was never really adopted or accepted in hematology practice. What is it about the hematology/oncology culture that prevented it from being more successful?

Dr. Wahl: That culture was 1 element, but there were others. This radioimmunotherapy clearly worked, and it was surprisingly nontoxic. At the time it was considered expensive at $\sim \$ 25,000$, and care delivery and reimbursement were challenging. The unlabeled CD20 antibody then turned out to be an effective therapy on its own. I also think that our approach was a little ahead of its time. Regardless, this effort defined a new paradigm, using patient-specific targeting and dosing that resulted in very predictable results with only modest toxicity. In October of this year, Novartis announced its planned acquisition of Endocyte (maker of ${ }^{177} \mathrm{Lu}-\mathrm{PSMA}-617$ and other radioligand therapeutics) for \$2.1 billion. So there is encouragement that big pharma is now seeing the economic benefit of theranostics. I'm proud to say that my colleagues and I helped to pave the way to some extent for other therapies to follow.

Dr. Czernin: What about your contributions to ${ }^{18}$ F-FDG PET imaging?

Dr. Wahl: When I went to the University of Michigan, I had a sense that PET was a powerful technique. It was the culmination of work that Michael Phelps and colleagues had done at Washington University. Mike and his collaborators had developed this technology and created widespread excitement. In the late 1980s at Michigan, we performed some animal studies with ${ }^{18}$ F-FDG in a large variety of tumors, showing the agent's great potential in tumor imaging. This built on pioneering work from Giovanni Di Chiro in brain tumors. In the late 1980s and early 1990s we started imaging cancers in patients, including melanoma and breast and lung cancers, using ${ }^{18} \mathrm{~F}-\mathrm{FDG}$ on early whole-body PET scanners brought to Michigan by David Kuhl.

The ${ }^{18}$ F-FDG PET method was not immediately appreciated. For example, we reported on the use of ${ }^{18} \mathrm{~F}-\mathrm{FDG}$ PET in women with breast cancer at the 1989 RSNA meeting. Five people were in the audience: 4 presenters and the projectionist. However, the images and additional studies in patients, especially in melanoma, showed remarkable target-to-background ratios. Localizing lesions was quite difficult with PET, and this is when we proposed and developed software methods to fuse PET and CT and also MR images as "anatometabolic" fusion images. I was particularly gratified when we fused PET and CT images to stage lung cancer. We showed in a prospective trial clear advantages for image fusion and for ${ }^{18} \mathrm{~F}-\mathrm{FDG}$ PET over CT for staging lung cancer. These early contributions on ${ }^{18} \mathrm{~F}-\mathrm{FDG}$ PET for staging in a wide range of cancers in humans, demonstrating that the PET method was superior and complementary to CT, were of particular relevance.

We also showed in preclinical and animal studies that ${ }^{18} \mathrm{~F}-\mathrm{FDG}$ was a marker of cancer cell numbers. This is when we started metabolic monitoring of breast cancer chemo- and hormone therapy - a very important development, because the concept of early response monitoring with PET did not exist at that time. Many studies were published subsequently using ${ }^{18}$ F-FDG PET for treatment assessments, not only in breast but also in other cancers such as lymphoma. I believe that we are still not using ${ }^{18}$ F-FDG PET as much as we should for treatment 
monitoring. The logical thing to do with ${ }^{18} \mathrm{~F}-\mathrm{FDG}$-avid tumors would be to acquire a baseline scan and then a 2- to 3-week scan after treatment initiation. Those patients with really outstanding responses are quite likely to need less therapy than those with a poor response. One could argue that a patient with a poor ${ }^{18} \mathrm{~F}-\mathrm{FDG}$ PET response should have his or her treatment changed if alternative therapies are available. Dose deintensification in those patients with exceptionally good responses would also seem reasonable. This would result in reductions in both side effects and costs. Great opportunities exist to achieve better risk-adapted patient management strategies based on quantitative PET imaging.

Dr. Czernin: Do you agree that treatments need to result in quick and robust reductions in tumor ${ }^{18} F-F D G$ uptake on PET? If these are not apparent on ${ }^{18} \mathrm{~F}-F D G$ PET imaging, the treatment cannot work. Favorable late glucose metabolic responses are essentially impossible.

Dr. Wahl: I generally agree, at least for chemotherapy and many targeted therapies. I don't think this is the case for checkpoint-inhibitor therapies, where flares in ${ }^{18}$ F-FDG uptake can sometimes occur early on in treatment, followed ultimately by a good response. We have a good tool for tumor response assessments with the PERCIST 1.0 criteria for treatment response. These simple criteria seem to have held up quite well to date. Having a uniform metric for assessing and tracking early response is very helpful, especially when it comes to comparing treatments across centers. The use of PERCIST 1.0 as opposed to anatomic imaging is a paradigm shift, but I am encouraged that more of the cooperative study groups are using PERCIST for treatment monitoring. Early monitoring will probably have an even greater role. One of the areas that has been underexplored with PERCIST is monitoring of bone-dominant disease, which is typically considered unmeasurable by the Response Evaluation Criteria in Solid Tumors. Disease in the skeleton is measurable with ${ }^{18}$ F-FDG PET/CT in breast cancer and other cancers-even in some aggressive prostate cancers. These are great opportunities, and we need clinical trials as well as to interface with specialists to include PERCIST in some of the trials that look at bone treatment responses. The RSNA QIBA initiative and the NCI QIN and Imaging Response Assessment Team initiatives for ${ }^{18} \mathrm{~F}-\mathrm{FDG}$
PET have helped move quantitative cancer treatment response assessments forward. I am glad to have been able to participate in these initiatives.

Dr. Czernin: We have to find ways to raise money to do clinical trials. There is not much industry interest, outside of the prostate cancer space, to support these trials.

Dr. Wahl: I concur. But it is encouraging to see major pharma investments in radiopharmaceutical therapies. I have often felt that if you examine the money society spends on health care across different sectors, the investment in imaging research is insufficient. Improved cancer patient management informed by imaging would likely reduce and not increase health-care costs.

Dr. Czernin: We have discussed nuclear medicine training, its interaction with radiology, and your contributions to $\mathrm{nu}$ clear medicine as translated into improved patient care. What are your priorities for the coming years, and how do you see the future of nuclear medicine?

Dr. Wahl: For the next few years, my primary role is to ensure that MIR is at the forefront of innovation, clinical care, education, and translation of technology to practice through commercialization and dissemination of knowledge. This includes progress in these multiple domains in nuclear medicine and careful integration of nuclear medicine methods with our other approaches, such as PET/MR imaging, as well as emphasizing quantitative imaging and therapy. With artificial intelligence and quantitation, I see radiology and nuclear medicine moving from qualitative and subjective tools to quantitative and objective ones. Our ability in nuclear medicine and radiology to provide real-time noninvasive phenotyping and multidimensional representations of patients, including imaging and genomic data, is expected to help us move personalized medicine forward. Appointments as chair are term limited, and time and health will determine how my post-radiology chair days will evolve. Because I regularly read out PET/CT, my expectation is that imaging and targeting the immune system in nuclear medicine will be opportunities for the foreseeable future, as well as continuing roles in education and mentorship.

Dr. Czernin: Richard, thank you very much for your time. 